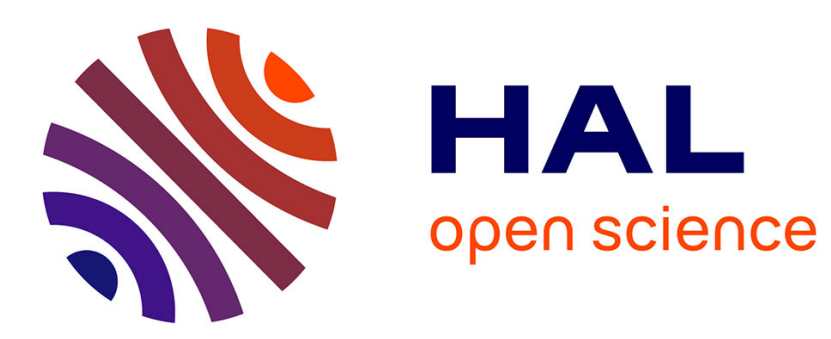

\title{
Du bureau au comité. La spécialisation du travail de l'Assemblée constituante (1789-1791)
}

Christophe Le Digol

\section{To cite this version:}

Christophe Le Digol. Du bureau au comité. La spécialisation du travail de l'Assemblée constituante (1789-1791). Cohen Antonin; Lacroix Bernard; Riutort Philippe. Les formes de l'activité politique, Presses Universitaires de France, pp.65-81, 2006, 9782130551508. halshs-02496091

\section{HAL Id: halshs-02496091 https://shs.hal.science/halshs-02496091}

Submitted on 13 Mar 2020

HAL is a multi-disciplinary open access archive for the deposit and dissemination of scientific research documents, whether they are published or not. The documents may come from teaching and research institutions in France or abroad, or from public or private research centers.
L'archive ouverte pluridisciplinaire HAL, est destinée au dépôt et à la diffusion de documents scientifiques de niveau recherche, publiés ou non, émanant des établissements d'enseignement et de recherche français ou étrangers, des laboratoires publics ou privés. 


\section{DU BUREAU AU GOMITÉ. LA SPÉGIALISATION DU TRAVAIL DE L’ASSEMBLÉE CONSTITUANTE (1789-1791)}

Christophe LE DigOL

Il est d'usage de considérer la compétition politique dans ses formes les plus visibles et les plus ostentatoires, c'est-à-dire celles qui mettent en scène les acteurs en conflit: joutes oratoires, affiliations partisanes, élections, etc. Ces figures de l'engagement public ont, par leur caractère symbolique, des effets sur la compréhension commune de l'action politique et des contraintes qui la régissent. En se focalisant exclusivement sur ces figures, l'analyste prolonge des constructions symboliques qui, issues des contraintes de l'action publique, peuvent constituer des obstacles à la compréhension de la formation et du fonctionnement de l'Assemblée constituante. Comparés à ces figures, les comités mis en place à partir de juin 1789 apparaissent secondaires. Constitués d'un petit nombre de députés chargés de s'occuper d'une question précise par l'Assemblée, ils remplacent rapidement les trente bureaux qui, à l'origine, divisaient tous les députés en petits groupes pour discuter des mêmes questions au même moment, avant d'engager le débat en assemblée plénière. Les analystes ont souvent tendance à considérer ces comités comme l'organe d'une fonction et, par conséquent, d'une division du travail que l'on peut expliquer en faisant appel aux nécessités du travail parlementaire. Que ce soit sous la forme d'impératifs pratiques qui imposent progressivement la division du travail la mieux adaptée à la fonction réelle ou supposée de l'Assemblée'. Que ce soit sous la forme d'un «besoin spontané » ou

1. André Castaldo, Les Méthodes de travail de la Constituante, Paris, PUF, 1989 ; Edna Hindie Lemay \& Alison Patrick (with a contribution by Joël Félix), Revolutionaries at Work 1789-1791, Oxford, Voltaire Foundation, 1996, p. 59 et s. 
d'un « mouvement naturel » qui suppose un ordre parlementaire idéel vers lequel tendrait par essence toute assemblée délibérante².

Ces approches mettent en scène des transformations dont les acteurs sont souvent ou absents ou relégués au rang de spectres dépourvus de caractéristiques sociales. En revanche, rapporter la formation de ces comités aux propriétés sociales des constituants qui les ont organisés permet d'identifier certains enjeux sociaux qui contribuent à faire de l'Assemblée un espace de valorisation et de reconnaissance de leur utilité sociale à la faveur d'un processus de spécialisation de son organisation du travail entre 1789 et 1791. Le passage du bureau au comité, comme mode dominant du travail d'Assemblée, objective, au cours de ces années riches de rebondissements politiques et sociaux, un ensemble de ressources sociales auparavant disponibles, mais jamais avec une telle ampleur, au sein d'une assemblée d'Ancien Régime ou d'une structure académique.

\section{Des États généraux à l'Assemblée constituante}

\section{Les bureaux}

Lorsque les députés du tiers état s'auto-instituent le 17 juin 1789 en Assemblée nationale constituante, se pose la question des formes dans lesquelles ils vont pratiquement s'assembler pour élaborer la Constitution. Dans l'urgence, les constituants sont contraints de donner une organisation à leur auto-institution. Dans un premier temps, elle va largement faire appel au mode consacré d'organisation des assemblées d'Ancien Régime : assemblées des notables et assemblées provinciales. Dans ces dernières, par exemple, les membres se réunissaient dans les bureaux alors qu'une fois assemblés en séance plénière, ils s'occupaient de lecture des rapports avant de rendre leur avis sous forme de vœux ${ }^{3}$. Ces assemblées d'Ancien Régime étaient divisées selon les cas en bureaux ou commissions, mais recouvraient

2. Joseph Barthélémy, Essai sur le travail parlementaire et le système des commissions, Paris, Librairie Delagrave, 1934, p. 19.

3. Pierre Renouvin, Les Assemblées provinciales de 1787, thèse de lettre, Paris, A. Picard, J. Gabalda, 1921, p. 152. 
des fonctions spécialisées ou non sans qu'aucun de ces termes ne renvoie a priori à un sens univoque d'une assemblée à une autre.

Dès le $1^{\text {er }}$ juin 1789, le député Pierre-François Aubry Dubochet propose d'organiser les états généraux en bureaux qui réuniraient les députés des trois ordres. Quelques jours plus tard, l'article 2 du «Chapitre Premier de la Police intérieure » du règlement provisoire indique que les bureaux remplacent la «division actuelle de l'assemblée par gouvernement». La division de l'assemblée en bureaux est donc la deuxième forme d'organisation adoptée par les députés. Les bureaux sont alors conçus comme des lieux où les députés débattent et relèvent leurs votes. Ils ne sont pas constitués selon des domaines déterminés ou, choix improbable au début du mois de juin 1789, en fonction des compétences de leurs membres. Lorsque Pierre-Victor Malouet propose de «donner à [chaque bureau] une matière particulière à discuter, à l'un les impôts, à l'autre le commerce, à celui-ci la justice, à celui-là l'agriculture, etc. ${ }^{4}$, il constate que sa proposition ne fait pas sens tant il est évident pour les constituants que les bureaux doivent «naturellement» s'occuper des mêmes objets en même temps, et successivement. Dès le 10 mai 1789, Mirabeau avait pourtant déjà proposé de s'appuyer sur la technologie des comités comme moyen d'accélérer le travail des communes et de promouvoir de cette manière les savoirs et le travail de ses membres. Le principe de constitution de ce comité, préconisé par Mirabeau, s'appuyait alors sur les divisions les plus légitimes dans l'Assemblée à ce moment-là : les députations provinciales.

Un mois plus tard, avec la division de l'Assemblée en bureaux, le principe de rassemblement par province disparaît. Tous les constituants sont affectés d'office dans un bureau selon une clé arithmétique. Ce procédé a l'avantage de «confondre heureusement toutes les provinces pour ne former qu'une même opinion \& un même esprit national. Elle servira à étouffer les cris discordants des privilèges \& des constitutions particulières des divers pays soumis à la domination française; elle fera disparaître enfin, par cette division des députés de la même province dans divers bureaux \& par la réunion de tous les esprits aux grands principes, cette variété si funeste des 
usages locaux, des administrations isolées $»^{5}$. Dans cette conjoncture, les bureaux sont perçus comme un dispositif pratique qui permet aux rapports interpersonnels de s'y nouer et de s'y développer dans le cadre d'une assemblée indivisée de 1200 personnes. C'est ce que souligne l'historien américain Timothy Tackett en évoquant le « rôle d'intégration » des bureaux nouvellement créés, dans un premier temps par et pour le tiers état, puis pour la noblesse et le clergé une fois ralliés au tiers état. Ils permettent à chacun des constituants, malgré leur "timidité », de s'investir dans le travail d'Assemblée indépendamment de leurs qualités oratoires ${ }^{6}$.

Formés au départ à partir de la division de l'Assemblée, les vingt puis trente bureaux sont destinés à fonctionner comme des fractions de celle-ci qui, après examen des motions soumises, délèguent la résolution des conflits et la production des décisions à l'ensemble des députés réunis en assemblée générale. Sous prétexte que ce «nouveau régime est contraire au règlement et divise l'Assemblée en trente confédérations délibérantes ", Armand-Gaston Camus dénie aux bureaux le droit d'élaborer des arrêtés avant qu'ils ne soient soumis à l'Assemblée ${ }^{7}$. En s'appuyant sur une configuration d'assemblée formée par les deux pôles assemblée générale/bureaux, les constituants ne semblent, dans un premier temps, envisager leur existence que sur un mode d'assemblée des voix et des hommes qui se suffirait à elle-même. L'adhésion aux formes d'un travail d'Assemblée socialement ajustées aux façons de voir des membres des ordres privilégiés favorise l'élaboration d'accords plus ou moins tacites sur les objets examinés et discutés.

\section{Des bureaux aux comités}

Avec la réunion des ordres s'ouvre une période d'incertitude liée au sens et aux fonctions de l'Assemblée, mais aussi aux appellations

5. Barrère de Vieuzac, Le Point du jour, ou résultat de ce qui s'est passé la veille à l'Assemblée nationale ( $\mathrm{n}^{\circ}$ 1-815, 19 juin $1789-1^{\text {er }}$ octobre 1791), Paris, Cussac, 26 vol., t. 1, jeudi 2 juillet 1789 , p. 89.

6. Timothy Tackett, Par la volonté du peuple. Comment les députés sont devenus révolutionnaires, Paris, Albin Michel, 1997, p. 136 et 155.

7. Archives parlementaires, t. VIII, 6 juillet 1789, p. 198. 
la désignant. S'il est convenu, le 7 juin 1789, que les matinées seront consacrées aux assemblées plénières et les après-midi au travail dans les bureaux, le règlement du 29 juillet 1789 prévoyait deux assemblées générales par semaine et une réunion quotidienne des bureaux. Alors que l'intégralité du travail est réalisé dans les bureaux appelés «bureaux de division », l'assemblée plénière, assemblée d'assemblages, semble avoir pour fonction la mise en scène légitimante, pratique et symbolique du travail collectif réalisé séparément et précédemment dans et par les bureaux.

En juin 1789, les membres assemblés du tiers état subvertissent la «tradition » de l'Assemblée des notables dans laquelle le roi prenait grand soin de refuser toute prise de décision en assemblée plénière, c'est-à-dire par un corps potentiellement souverain et alternatif à sa personne. Ici, la substitution a lieu, l'assemblée plénière met en place les conditions qui permettent la captation et l'incarnation de la souveraineté dans le corps réuni des députés. Elle entérine les décisions prises dans les bureaux et sous leur contrôle. Dans le même temps, cette nouvelle organisation est de facto auto-instituante et autoconvocatrice d'elle-même. Dans cette conjoncture d'intensification des luttes entre le roi et les constituants, l'invocation et la mise en scène d'une souveraineté qui s'incarne dans le corps réalisé des députés réunis en assemblées plénières est au principe de leur multiplication au détriment du travail des bureaux dont la réunion va être rapidement reléguée en soirée comme le regrettera quelques mois plus tard Jean-Joseph Mounier : "Les bureaux offraient surtout une grande ressource. C'était là que, dégagés de tout ce qui excite la vanité, n'ayant plus les applaudissements des spectateurs à désirer, les marques d'impression à craindre, n'ayant point de discours à prononcer pour les faire insérer dans les gazettes, on préparait, avec l'attention la plus scrupuleuse, les diverses questions qui devaient être traitées dans l'Assemblée, et que beaucoup d'hommes modestes opposaient la froide raison et l'expérience à la chaleur des idées prétendues philosophiques. Mais ces derniers moyens, propres à corriger les vices de la méthode adoptée, furent bientôt détruits. Beaucoup de motions ne furent point renvoyées, beaucoup de questions importantes n'ont pas été soumises à la discussion de trois jours; et enfin, le lendemain du jour [où] le règlement fut admis, on soutint que 
l'enthousiasme patriotique s'affaiblissait dans les bureaux: on demanda qu'il y eût une assemblée générale tous les matins; bientôt il y en eut deux par jour, et les bureaux devinrent inutiles. J'ai beaucoup à me reprocher de n'avoir pas résisté avec énergie aux moyens mis en usage pour anéantir les bureaux. $»^{8}$

Selon Camille Desmoulins, la responsabilité de l'abandon des bureaux doit être rejetée sur Isaac-René Le Chapelier, président à ce moment-là de l'Assemblée nationale, qui, après l'adoption du règlement le 29 juillet 1789, aurait multiplié les réunions de l'assemblée plénière, empêchant ipso facto le bon fonctionnement des bureaux. Progressivement dessaisis de leurs fonctions d'examen et de discussion préalable des motions, les bureaux ne conservent que leur fonction élective, notamment des membres de certains comités. Les premiers comités sont d'ailleurs formés à partir des bureaux. En ce qui concerne le comité de division du travail par exemple, chargé de préparer un plan d'élaboration de la Constitution, chaque bureau a pour mission de choisir un membre en son sein pour constituer un comité de trente députés. Les procédures initiales de formation des comités expliquent notamment les importants effectifs de députés composant ces premiers comités. Le temps passant, le nombre de députés composants les comités va decrescendo, allant de pair avec la nomination de leurs membres par l'assemblée plénière et non plus par les bureaux.

Le mode d'assemblée envisagé dès les premiers jours de la Constituante s'inscrit dans une démarche légitime qui dévolue la capacité de décision à une autorité extérieure, le roi. En rendant obsolètes les formes antérieures de débats - auxquelles la forme "spontanément » adoptée des bureaux correspond -, qui n'imposaient pas à leurs participants de produire une décision au terme de ceux-ci, l'autoproclamation du 17 juin 1789 en transforme progressivement les finalités pratiques. Cette auto-institution n'est pas seulement un coup de force symbolique qui opère une rupture avec l'ordre symbolique ancien. Les constituants initient du même coup une configuration qui leur impose

8. Jean-Joseph Mounier, «Exposé de ma conduite dans l'Assemblée nationale », Orateurs de la Révolution française, t. I : Les Constituants, textes établis, présentés et annotés par François Furet et Ran Halévi, Paris, Gallimard, 1989, p. 926-927. 
de produire des réponses pratiques, de régler et de coordonner des exigences sociales et politiques qui, formulées dès la préparation des états généraux, convergent dorénavant vers l'Assemblée. Ils ne forment pas seulement un souverain symbolique mais aussi un souverain décisionnel.

\section{L'emprise des comités}

Ces nouvelles contraintes ne sont pas sans conséquences sur l'organisation du travail dans l'Assemblée. Les comités, dont les quatre premiers (les comités de rédaction, de règlement, des subsistances et de vérification) sont créés deux jours après l'autoproclamation de l'Assemblée nationale constituante, forment, contrairement aux bureaux, une structure spécialisée d'examen et de préparation des questions, composée d'un petit nombre de constituants devant prendre en charge les problèmes qui se posent et s'imposent à l'Assemblée. Leur structure favorise et accélère les prises de décision d'une assemblée qui se veut délibérative. Les comités se mettent en place au fur et à mesure que les députés acquièrent du pouvoir sous l'effet de l'accumulation des soutiens tant provinciaux que parisiens. Pris dans les luttes pour la redéfinition des formes légitimes de la représentation, les constituants mettent en place ces organes de captation des capacités d'action comme un moyen d'affirmation de leur utilité politique. Ils instaurent ainsi les conditions de la pérennisation de l'Assemblée. Dans cette logique, environ trentecinq comités seront créés entre le 19 juin 1789 et le printemps 1790 . Ces comités se forment au coup par coup, dans la logique pratique des problèmes politiques qui se posent aux constituants, mais aussi dans la logique politique qui consiste à autonomiser et à ériger des problèmes pratiques en enjeu de luttes entre les députés de l'Assemblée. Une fois leur nombre stabilisé, le flux de nominations et de transferts dont ils font l'objet est le signe qu'ils deviennent le centre de gravité du travail de la Constituante.

Les mois passant, l'emprise croissante des comités et de leurs membres sur le travail de l'Assemblée ne va pas sans susciter des réactions de la part de tous ceux qui, attachés à une définition de l'Assemblée incarnée par la souveraineté de son assemblée plénière, 
critiquent la subordination croissante de l'Assemblée à leurs travaux. «Dans l'abandon de l'Assemblée actuelle à ses comités, on ne peut apercevoir qu'une sorte de lassitude, ou d'envie de terminer l'œuvre de la Constitution, aux dépens même de sa perfection ; mais ces dispositions effraient les bons citoyens ; ils se rappellent encore ces premiers moments de vigueur, et cette inquiétude vigilante qui consacra les premiers travaux de l'Assemblée nationale; ils se rappellent les belles discussions dont ont émanées nos plus sages lois; alors ces comités n'étaient que des conseils, ils sont aujourd'hui ses ministres. ${ }^{9}$ Ils dénoncent aussi de manière récurrente la violation de l'article du règlement qui interdit d'appartenir à plus d'un comité, accusant les députés qui ne le respectent pas de composer une nouvelle aristocratie ${ }^{10}$. Ils dénoncent enfin l'autonomie croissante des comités qui rendent des avis et des décisions sans accord préalable de l'Assemblée. Le 26 décembre 1789, sur une proposition d'Hébrard, transformée en décret par Adrien Duport, elle prend la décision qu'aucun comité ne pourra rendre public son avis sans son consentement. Mais, le 31 janvier 1791, une motion d'Antoine-Balthazar d'André appelant à ce que «les comités ne puissent plus donner de décisions sur les décrets de l'Assemblée nationale » est repoussée sous prétexte que ces «décisions sont si utiles dans un grand nombre de départements, que les lois décrétées trouveront des obstacles dans leur exécution si l'on ne les a pas $»^{11}$.

Les travaux des différents comités structurent à tel point ceux de l'Assemblée que les constituants, réunis en assemblée générale le 30 mars 1790, se plaignent que les délibérations de l'Assemblée souffrent du retard des travaux menés par les comités. À cette occasion, Philibert de Fumel-Montségur les excuse en soulignant que les «comités font le travail de 1200 personnes; il n'est pas étonnant qu'ils soient surchargés et ne puissent faire face à tout $»^{12}$. Les problèmes qu'éprouvent les députés à coordonner le travail entre les comités et les séances plénières peuvent laisser penser que l'Assem-

9. Kersaint (de), «Des classes des gens de mer, ou nouvelles réflexions sur la plus importante de nos institutions maritimes ", Journal de la Société de 1789, n IX, 29 juillet 1790, p. 20-21.

10. Foucault de Lardimalie, Archives parlementaires, t. XI, 21 janvier 1790, p. 266.

11. Archives parlementaires, t. XXII, 31 janvier 1791, p. 590.

12. Archives parlementaires, t. XII, 30 mars 1790, p. 443. 
blée se trouve progressivement subordonnée aux comités. Ils expriment surtout les difficultés qu'éprouvent les constituants face à la complexité croissante d'une Assemblée, c'est-à-dire face à l'interdépendance grandissante entre des espaces d'Assemblée de plus en plus spécialisés.

Outre qu'il assure la prééminence d'une forme d'organisation nouvelle, le déplacement du centre de gravité des bureaux vers les comités accroît l'influence d'un ensemble de députés dont les compétences et les savoir-faire sont sollicités dans ces comités. Ils acquièrent une autorité que l'organisation en bureaux ne leur conférait pas : ces derniers mettaient en circulation des opinions dont l'assentiment n'était pas fondé sur une connaissance précise des questions examinées mais sur un exercice de la parole dont l'enjeu restait la valorisation de celui qui l'exerce au sein d'une petite assemblée de pairs, dotés ou non des mêmes dispositions que le locuteur, mais aussi sensibles que lui à l'intelligence et au trait d'esprit que peut susciter le traitement d'une question, tel qu'il est coutume de l'exercer dans une académie de province ou dans un salon. «On nous a divisés par bureau. Je suis avec le chevalier de Boufflers, qui a de l'esprit, de l'amabilité. Dans le fond, tout cet arrangement m'est très indifférent : j'y gagnerai même, car j'aurai nécessairement plus d'influence dans l'assemblée générale que dans l'assemblée partielle de la noblesse. Mon esprit doux et conciliant, la facilité que j'ai de parler sur toutes sortes de sujets, et d'aller, dans les conversations particulières, chercher le mérite des gens avec lesquels je cause, de leur donner le plaisir de le démontrer, me gagneront promptement l'amitié et la bienveillance de la plus grande partie des membres du Tiers. $»^{13}$ Les bureaux valorisaient une capacité à prendre la parole et un «amateurisme » désintéressé et éclairé que l'orateur et son auditoire devaient manifester à cette occasion.

Ces qualités sont à l'opposé des exigences de travail et de constitution de dossiers qui fondent dans l'urgence la nécessité des comités. La création de ces derniers autour d'objets précis mobilise au contraire tous ceux qui, dotés à un titre ou à un autre de ressources

13. Charles-Élie de Ferrières, Correspondance inédite (1789, 1790, 1791), publiée et annotée par Henri Carré, Paris, Librairie Armand Colin, 1932, p. 78. 
sociales spécifiques, peuvent concourir dans cette conjoncture d'urgence à structurer cette nouvelle organisation et ses productions. Une forte logique de spécialisation est à l'œuvre dans ces comités. Elle concourt à favoriser l'apparition au premier plan de ceux qui avaient précédemment quelques compétences effectives à propos des tâches que l'Assemblée leur a confiées, même si ces compétences ne sont pas systématiquement au principe de leur nomination. Cette dépendance du travail des comités vis-à-vis de quelques constituants que leurs activités et leurs compétences rendent indispensables constitue l'une des raisons pour laquelle l'Assemblée abandonne, dès la fin de l'année 1789, leur renouvellement mensuel, initialement prévu.

\section{Les fonctions sociales de l'Assemblée constituante}

\section{L'engagement dans les comités}

Le nouveau dispositif institutionnel, dont les comités sont l'une des deux pièces maîtresses, requiert et mobilise des dispositions sociales qui, en s'imposant au sein de cette structure de travail spécialisée, font dépendre le travail lui-même et les décisions préconisées et adoptées de certaines dispositions sociales préalablement acquises et incorporées par les députés, lesquelles disqualifient de facto tous ceux dont l'essentiel du savoir-être et du savoir-faire est lié à un exercice de la parole désintéressée. Les comités requièrent des savoirs et des savoirfaire spécifiques : juridiques (comités de constitution, de rédaction, des décrets, des droits féodaux); financiers (comités des finances, des douze, de l'extraordinaire, de l'imposition ou des contributions publiques, des monnaies) ; économiques (comités des subsistances, d'agriculture et de commerce), etc. La mobilisation de savoir-faire destinés à satisfaire dans l'urgence à des exigences pratiques porte ainsi au premier plan tous ceux qui, dans leur parcours social antérieur, ont développé un intérêt pour des types de savoirs et de compétences comme seul moyen ou de maintenir leur rang social ou d'accéder à un rang plus élevé.

Leur trajectoire sociale les avait conduits à s'écarter d'une logique nobiliaire du désintéressement sous peine de parfois subir un certain 
déclassement social. Elle leur confère désormais valeur et honneur ainsi qu'une utilité sociale jamais auparavant reconnue et instituée avec une telle ampleur en devenant dans cette conjoncture les acteurs structurants de ce nouveau dispositif institutionnel. L'inégale répartition des dispositions sociales dorénavant requises explique que seuls 496 députés, soit environ $40 \%$ de l'ensemble des constituants, vont occuper les 832 postes électifs créés par les comités. Et 187 d'entre eux, soit $14 \%$ du total, détiennent $63 \%$ de ces postes. En outre, nombre de ces constituants font partie des principaux orateurs de l'Assemblée et en monopolisent les fonctions de secrétaire et de président. Après le 30 juin 1789, les trois quarts des présidents de l'Assemblée sont élus parmi les constituants ayant été nommés dans au moins trois comités ${ }^{14}$.

Appréhender les variations de l'engagement des constituants dans les comités à travers la catégorie instituée de l'ordre, c'est-à-dire en fonction d'une logique fondée sur le rang social, suppose de comprendre ce que recouvrent ces appartenances en termes de spectre de trajectoires et d'acquisitions de savoirs et de savoir-faire. Les membres du tiers état, composé notamment pour $49 \%$ de détenteurs d'offices (un minimum de connaissances juridiques est requis pour exercer les fonctions publiques impliquées par la possession d'offices) et pour $23 \%$ d'avocats ${ }^{15}$, ont des manières d'être et de faire qui s'appuient davantage sur des logiques d'accumulation de ressources spécifiques (droit, médecine, science, négoce, etc.), dont dépend en grande partie l'autorité de leur position sociale, que les membres des deux premiers ordres. Ces compétences, socialement dévalorisées dans une économie des biens symboliques dominée par un sens nobiliaire des valeurs, trouvent néanmoins dans le dispositif centré autour du travail des comités l'occasion d'une valorisation sociale inédite. Aussi 308 d'entre eux occupent-ils 512 des postes disponibles dans les comités.

14. Edna Hindie Lemay \& Alison Patrick (with a contribution by Joël Félix), Revolutionaries at work..., op. cit., p. 62.

15. Edna Hindie Lemay, "Valeurs nouvelles et leur pratique dans des discours des députés juristes à l'Assemblée constituante ", La Révolution et l'ordre juridique privé - rationalité ou scandale, actes du colloque d'Orléans 11-13 septembre 1986, t. I, présentation de Michel Vovelle, Paris, PUF, 1988, p. 220. 
Ces dispositions ne sont néanmoins pas réservées aux seuls membres du Tiers. On peut les trouver à l'œuvre chez les membres de la noblesse (103 membres de comités pour 199 postes occupés) et, à un degré moindre, chez le clergé (93 membres de comités pour 121 postes occupés). Dans ce dernier ordre, seuls huit ecclésiastiques cumulent 3 places (ou plus) dans les comités (28 postes en tout). Si l'on considère les propriétés sociales de ces ecclésiastiques - celui qui cumule le plus grand nombre de postes (7) est Charles-Maurice de Talleyrand-Périgord, évêque d'Autun -, on s'aperçoit qu'ils sont dotés de propriétés sociales peu partagées par les membres de leur ordre et liées à des trajectoires peu communes. Le clergé a ainsi la caractéristique de souffrir d'un investissement à la fois faible et remarquablement dilué dans la structure des comités. Les nobles, au contraire, sont 28 à cumuler trois places (ou plus) occupant au total 100 postes (sur les 199 occupés par la noblesse) ${ }^{16}$. À propos de ces derniers, Alison Patrick observe que «the greater the concentration of office in the hands of individuals, the more likely it was that those individuals would be drawn from the (depleted) ranks of the nobility ${ }^{17}$. La relative désaffection des nobles de cour est pour partie liée à leur fréquentation de la cour qui offre un accès privilégié à des positions et, par conséquent, à des ressources concurrentes de celles que l'Assemblée accorde ; et, pour une autre partie, au désajustement progressif entre les dispositions sociales des députés nobles et celles dorénavant requises et valorisées par l'Assemblée.

En fait, plus les ressources sociales nouvellement pertinentes au sein de l'Assemblée sont rares chez les membres d'un ordre (clergé et noblesse), plus le phénomène de concentration des postes est élevé en son sein. Plus les ressources sociales pertinentes sont partagées entre les membres du même ordre (le tiers état), plus la concentration des postes diminue. Ces correspondances mettent en évidence un phénomène de concurrence différemment proportionnée selon les ordres autour des ressources sociales devenues pertinentes à la faveur de l'institutionnalisation du travail en comités. Le dispositif institutionnel

16. Edna Hindie Lemay \& Alison Patrick (with a contribution by Joël Félix), Revolutionaries at Work..., op. cit., p. 63.

17. Alison Patrick, "The Second Estate in the Constituent Assembly, 1789-1791 », Journal of Modern History, juin 1990, p. 238. 
que dessinent les comités privilégie donc tous ceux qui, membres du Tiers ou tournés vers lui, possèdent quelques savoir-faire et compétences ajustés aux nouvelles exigences pratiques.

L'inégalité de participation au travail mené au sein des comités n'exprime pas seulement une relation structurée et structurante entre des propriétés sociales inégalement réparties et incarnées, et leur objectivation sous la forme d'un nouveau mode d'organisation du travail d'assemblée. Elle traduit aussi l'adhésion à des univers symboliques fort différents. Dans son ouvrage sur l'Assemblée constituante, Timothy Tackett explore l'« esprit » des députés révolutionnaires par l'intermédiaire du rapport au monde d'Ancien Régime, des manières de penser et des formes de croyance engagées dans leur action, lesquelles, si l'on en croit l'auteur, seraient susceptibles de structurer de manière commune leur action par-delà les différences sociales séparant ces députés. Si dix députés seulement peuvent être selon lui qualifiés de philosophes dans le sens du XVIII ${ }^{\mathrm{e}}$ siècle, les membres de loges maçonniques représentent environ $20 \%$ des députés : trois cinquièmes appartiennent au tiers état, un tiers à la noblesse et moins d'un dixième au clergé. L'analyse des publications des députés révèle aussi de notables différences dans le choix des sujets abordés : comme on pouvait s'y attendre, les membres du clergé écrivent $90 \%$ des ouvrages de religion et de théologie. Les nobles, pour leur part, rédigent surtout des ouvrages de littérature et de poésie (plus du tiers de leur production) et n'écrivent presque rien sur les questions de droit. En revanche, les députés du tiers état publient des ouvrages sur les sujets les plus variés (droit, histoire, économie politique, géographie, etc.).

Les données biographiques disponibles sur les ecclésiastiques cumulant au moins trois postes dans les comités laissent entrevoir l'existence de pratiques de lecture et d'assemblée, voire une certaine familiarité avec des espaces de sociabilité comme la franc-maçonnerie, qui peuvent expliquer leur disposition à s'engager davantage que leurs pairs dans le travail d'Assemblée ${ }^{18}$. La dynamique de construction du

18. C'est le cas notamment du curé de Cery, Jean-Baptiste Massieu, membre de trois comités, qui avait été le précepteur des frères Lameth, futurs constituants, puis professeur de rhétorique au collège de Vernon. Ces brèves données biographiques laissent supposer la maîtrise d'un matériel symbolique qui va bien au-delà des connaissances théologiques généralement détenues par le bas clergé. 
travail des comités sélectionne parmi les ordres privilégiés un personnel qui, issu de différents univers sociaux et symboliques, maîtrise les instruments symboliques suffisants pour réajuster manière d'être et mécanisme incorporé d'interaction au travail en comité. Les comités, en réalisant au sein de l'Assemblée constituante une objectivation inédite de propriétés sociales, portent au premier plan un ensemble d'acteurs dont la puissance sociale dominée l'était d'abord symboliquement par la nature du rang de ceux qui l'incarnent. Leur capacité à inventer et à mettre en forme une nouvelle institution, si elle peut étonner, n'est néanmoins que la réalisation dans une conjoncture inédite et ouverte de la puissance sociale du tiers état dont Sieyès avait proclamé qu’il était « tout $»^{19}$.

\section{Enjeux de formes et coordination du travail d'Assemblée}

Si, du point de vue de son assemblée plénière, l'Assemblée peut paraître subordonnée au travail des comités, ces derniers ne sont pourtant pas des organes souverains et inaccessibles aux enjeux qui structurent les décisions prises par la Constituante. Au contraire, ils sont censés être subordonnés aux enjeux et aux objectifs dégagés par l'assemblée plénière. Il n'est donc pas surprenant que la nomination de leurs membres devienne très rapidement un enjeu de luttes entre les différentes factions de l'Assemblée : «Les Jacobins se rassemblent trois fois la semaine. Leur séance est véritablement \& en un mot propre la répétition de la séance du lendemain à l'Assemblée nationale. Là se proposent \& se discutent les motions, se rédigent les décrets, se nomment les présidents \& secrétaires de l'Assemblée nationale, les membres des différents comités ; \& comme ils ont la majorité, tout ce qu'ils ont délibéré la veille est chose conclue $\&$ décrétée. $»^{20}$

La capacité des comités à prendre en charge les enjeux qui structurent les prises de position au sein de l'Assemblée et à assumer un rythme de travail conséquent est le produit des rapports de force qui

19. Emmanuel Sieyès, Qu'est-ce que le Tiers État?, Paris, PUF, 1989.

20. Révolutions de France et de Brabant, n 10, t. I, p. 438-439. 
se tissent entre les membres composant chaque comité. Le comité ecclésiastique est à ce titre intéressant car les tensions qui l'animent ont provoqué la paralysie de ses travaux : «Ce comité était, comme je l'ai dit, composé de membres contraires en opinion. Ceux qui avaient été pris des deux ordres alors supprimés y tenaient encore par tous leurs préjugés et toutes leurs prérogatives. $»^{21}$ La composition de ce comité comme le problème pratique au fondement de sa formation assurent un relatif équilibre entre les constituants inscrits dans l'entreprise de redéfinition de l'ordre ancien et ceux dont la position dans cet ordre ancien et l'intériorisation du rapport à cet ordre sous la forme de dispositions sociales et mentales n'encouragent pas à faire advenir l'ordre nouveau que les premiers appellent de leurs vœux. Les premières mesures que l'Assemblée décrète en matière religieuse (décret du 2-4 novembre 1789²2) entraînent l'évêque de Clermont, l'évêque de Luçon, Charles-Léon de Beaujeu de Bouthillier, ÉtienneFrançois Sallé de Choux, Suzain-Gilles Vaneau, François-Marie Grandin, Jean-Jacques de La Lande et l'abbé de Montesquiou, tous membres du comité ecclésiastique, dans une opposition qui vise à conserver en l'état l'organisation et les conditions matérielles qui fondent la puissance temporelle et spirituelle du clergé. Leur opposition entrave si bien le travail du comité que les députés réunis en assemblée plénière décident en février 1790 de faire nommer quinze députés supplémentaires pour mettre fin à leur obstruction. Au-delà du caractère anecdotique de ces nominations, cette décision de l'Assemblée révèle la mise en place d'un ordre d'assemblée qui assujettit chacune de ses subdivisions à la réalisation d'une Constitution conforme aux intérêts de la majorité des députés qui la composent.

Les séances publiques que les députés mettent en scène ont notamment pour fonction de mettre symboliquement en forme et de rendre les propriétés sociales, redéfinies par le travail en comités, conformes aux nouvelles croyances politiques et sociales que les députés fabriquent au gré des luttes qui les opposent au pouvoir royal ainsi que les uns aux autres. Elles concourent ainsi à produire

21. Pierre-Toussaint Durand-Maillane, Histoire apologétique du comité ecclésiastique, Paris, chez F. Buisson, Imprimeur-Libraire, 1791, p. 6.

22. Décret du 2-4 novembre 1789 qui met les biens ecclésiastiques à la disposition de la nation. 
les nouvelles justifications qui accompagnent et consacrent le travail de redéfinition des positions politiques comme des positions sociales. Cette mise en scène légitimante, tant de l'action des députés que du résultat de leur action, ne peut pas seulement se concevoir, comme on le fait souvent, comme une mobilisation de principes philosophiques abstraits maniés par des « discoureurs » aussi brillants que vains. Il faut réfléchir au rôle qu'occupent ces innombrables discours dans une économie d'Assemblée de plus en plus complexe : du point de vue du mécanisme de production de l'autorité de l'Assemblée d'une part ; du point de vue du mécanisme de justification et de conformation symbolique du travail social que les députés effectuent sous couvert de leur activité d'Assemblée, d'autre part.

Ce travail d'ajustement du travail social réalisé en comités aux principes politiques au nom desquels les constituants prétendent agir permet de présenter symboliquement comme conformes des stratégies et des mises en forme sociales parfois très différentes les unes des autres. Loin d'être aussi inutiles que les dénonciateurs de l'Assemblée le suggèrent dans la logique pratique de disqualification du travail d'Assemblée, les discours d'Assemblée remplissent donc un rôle éminent dont les députés ne peuvent se passer. Dans ses divisions pratiques et symboliques, l'Assemblée est un dispositif complexe au sein duquel chaque forme de travail prend sens relativement aux autres sans qu'il ne soit jamais possible de les isoler pratiquement. Un ordre d'assemblée ne s'organise pas naturellement sous l'effet d'un commun accord entre les membres de l'Assemblée ou d'une nécessité historique qui s'imposerait à tous. Il est un enjeu de luttes entre les membres de l'Assemblée qui cherchent à consolider leurs points de vue en imposant les formes les plus appropriées aux finalités pratiques dans lesquelles ils sont inégalement pris. La configuration séances publiques/comités offre aux députés investis dans un travail de construction et de promotion de l'Assemblée nationale les moyens de contrôler et de coordonner le travail effectué comme son rythme d'une manière plus efficace que ne le faisait la configuration précédente assemblée plénière/bureaux, laquelle était fondée sur l'indépendance des bureaux considérés comme unités délibératives souveraines.

Cette différenciation des espaces et du travail d'Assemblée prend sens dans la logique des décisions législatives qu'elle s'est donnée pour 
obligation de produire. L'Assemblée est un mécanisme complexe de production des décrets : d'identification et de définition de problèmes sociaux, d'examen de ces problèmes en comités, d'élaboration et de présentation de solutions à ces problèmes sous la forme de rapports et de projets de décret(s) lus et discutés en séances publiques, et éventuellement d'approbation de ces projets en décret(s), une fois remaniés dans ces séances publiques qui leur confèrent le sceau de l'universalité et de la «Nation». Il s'agit de manifester, dans l'approbation collective des députés, l'intérêt et l'utilité que certaines ressources sociales présentent pour la «Nation », c'est-à-dire pour l'ensemble des groupes en position de redéfinir à leur profit les interdépendances sociales en transformant les intérêts particuliers qu'ils ont en commun en intérêt général pleinement reconnu et accepté par une "Nation » dont ils sont devenus les porte-parole autorisés et dont ils mettent en scène l'existence.

L'inégale mobilisation des ressources sociales dans l'Assemblée engage, comme le suggèrent certains indices, beaucoup plus que la construction d'un ordre d'Assemblée. Elle a partie liée avec ce que sont ces ressources hors de l'Assemblée, c'est-à-dire avec un état de la structure sociale qui définit et garantit la valeur relative de ces ressources sociales. Elle a aussi partie liée avec le travail de mise en forme nouveau et centralisé de ces ressources. Ce faisant, les constituants effectuent un travail d'homogénéisation et d'universalisation symbolique et pratique de ressources sociales qui ne possédaient pas, sous l'Ancien Régime, l'universalité que confère une reconnaissance centralisée, c'est-à-dire par une instance reconnue comme centrale, ayant la capacité d'imposer les formes légitimes dans lesquelles elles sont reconnues à/par l'ensemble des groupes dépendant du ressort du gouvernement royal. Par le truchement du travail effectué en comités, les constituants concourent à redéfinir les formes qu'adoptent ces ressources, à les mettre en conformité les unes par rapport aux autres, c'est-à-dire à produire les conditions de la reconnaissance et de la circulation ajustées aux dispositions sociales qui mobilisent à la fois les députés présents à l'Assemblée et les agents sociaux extérieurs à l'Assemblée, possédant des propriétés homologues à celles des députés. 